\title{
AN APPLICATION OF THE ANALYTIC HIERARCHY PROCESS (AHP) FOR LOCATING A DISTRIBUTION CENTER
}

\author{
Flávio de Almeida Galvão Jr. \\ flavio.galvaojr@terra.com.br \\ Claudio Barbieri da Cunha \\ cbcunha@usp.br \\ Nicolau D. Fares Gualda \\ ngualda@usp.br \\ Departmento de Engenharia de Transportes - Escola Politécnica - Universidade de São Paulo - Brasil \\ Caixa Postal 61548 - CEP 05424-970 - São Paulo - SP - Brasil
}

\begin{abstract}
Keywords: AHP, Site Selection, Location, Distribution Center, Logistics

\section{Introduction}

Site selection for the most different purposes has been widely discussed in different forms, but generally restricted to tangible aspects. Mathematical modeling aimed at maximizing or minimizing an objective function, generally associated to total profit or cost, has been a commonly used approach. The decision on where to locate facilities involves considering several criteria and attributes with different influence levels in the final outcome, whatever it may be, including the need to contemplate the quantitative and qualitative issues in this analysis, what increases the complexity of the problem.

One relevant contribution of this study consists in the proposition and application of a typical check list, as generic as possible, that can be useful in a wide range of site selection problems utilizing multicriterial methodologies as support to decisions, such as the AHP. A case study involving a site selection for a distribution center of a company in the non-durable consumer goods sector is considered. It was solved using the Expert Choice software, which encompasses the AHP concepts.
\end{abstract}

\section{Modeling the facility location problem}

Mathematical models tend to reflect and represent partial aspects of real cases, as pointed out by Pidd (1999), otherwise becoming extremely complex to solving. This is particularly true concerning facility location problems. Along the past few years, and with a greater emphasis as from the 1970s, new tools for solving complex problems have been discussed and proposed to overcome the difficulties faced by traditional optimization models based on mathematical programming to adequately represent and solve complex problems such as the facility location ones.

Lin and Tu (2000) state that the facility location problem is complex and that the AHP can, effectively and appropriately, deal with the qualitative and quantitative factors in a multiple criteria decision environment, thus being an important tool to synthesize in scenarios and to produce a diagnosis that allows the decision maker to understand the inter-relational behavior of the systems that form decision.

The environment for selecting the location of the distribution center configures a complex problem with different qualitative and quantitative attributes, both tangible and intangible, that systemically interact and have to be dealt with concomitantly. The lack of knowledge on certain attributes that would normally be 
tangible requires them to be considered as intangible, thus increasing the complexity of the decision making process. The scenario described above coincides with several strategic decision making situations, in which little is known about what is to be decided upon, little is to be spent to support the decision process and the decision must be reached in a short period of time.

\section{The use of a checklist and AHP for site selection}

Gualda (1995) discusses aspects of the location theory, stressing its multidisciplinary character and calling attention to different factors to be considered and weighted in treating real world location problems. These range from factors contemplated in the company strategic planning, in its master planning, in its production planning, as well as in its socio-economic environment.

A typical checklist encompassing items relevant to site selection was elaborated. It contemplates nearly 100 parameters to be assessed such as those related to inputs (water and effluents, gas, electric power, transport of raw materials and products), market and environmental aspects, urban occupation and housing, human resources, life quality, taxes, etc. It is generic in nature and should be adapted to the type of premise to be evaluated and implemented, with the necessary adjustments to the respective intrinsic characteristics of the problem being considered. The list aims to encompass different attributes related to the effectiveness of the facility location.

Environmental constraints, technological capacitation and union relationship have recently exerted greater influence on strategic decision. These aspects have proven to be difficult to be considered in the traditional methods, such as the discounted cash flow one, due to the need to convert them into monetary values.

\section{Application of AHP to a logistic problem of site selection for a distribution center}

The AHP method was applied to select the most adequate location to implement a distribution center for a given company in the non-durable consumer goods sector whose products require special packaging and handling due to their fragility. The three candidate sites for implementing the distribution center are located in the outskirts of the São Paulo metropolitan region, in Brazil

The attributes proposed in the checklist were adjusted and implemented hierarchically in the Expert Choice software. The resulting inconsistency index of the judgments was of about 0.01 , which can be considered as good enough, giving the large amount of variables evaluated. A sensitivity analysis concerning the first level of the hierarchy was also performed, involving the simulation of some variations on chosen attributes.

\section{Conclusions and final remarks}

AHP has shown to be a suitable tool to support location decision making, mainly in the initial phase of the evaluation process, in which there is a lack of some data required by a more sophisticated modeling approach. It allowed the identification of the most adequate location for implementing the Distribution Center in the studied case.

The proposition of a comprehensive checklist can be seen as a relevant contribution of the present research. It can be useful in a wide range of site selection problems, mainly those related to multicriterial methodologies to support decision. In particular, for application of AHP to location problems.

The proposed checklist is somehow extensive and may not be fully required in some cases since its evaluation may be time and effort consuming. However the large number of items in the checklist provides guidance for selecting relevant attributes influencing site selection in specific cases.

It should be noticed that a single hierarchic structure was organized to take into consideration both costs and benefits. Separate structures could be considered for costs and benefits. However, this split approach would not add to the quality of the solution, given the quality of the available data. 